\title{
50 Besonderheiten bei Diäten
}

\author{
(c) Springer-Verlag GmbH Deutschland, ein Teil von Springer Nature 2018 \\ D. Mathias, Fit und gesund von 1 bis Hundert \\ https://doi.org/10.1007/978-3-662-56307-6_50
}

Statistisch betrachtet verspeisen Deutsche tagtäglich 130-150 g Fett, aber je nach dem individuellen Kalorienbedarf sind nur rund $90-110$ g auf Dauer gesundheitlich vertretbar. Eine solche Begrenzung einzuhalten fällt jedoch oft schwer. Denn in vielen Lebensmitteln oder im Kantinenessen ist der Fettgehalt häufig nicht deklariert. Und eine Menge Fett ist dort versteckt, wo man es gar nicht vermutet, allein bis zu $8 \%$ im Vollkornbrot. Wir essen aber nicht nur zu viel Fett, wir verspeisen überwiegend tierisches Fett und mit den darin vorkommenden gesättigten Fettsäuren das falsche Fett.

$10 \mathrm{~g}$ Fett (gesättigte Fettsäuren) sind jeweils enthalten in:

- 2/3 Croissant

- 2 Joghurt-Riegel à $25 \mathrm{~g}$

- 16 Kartoffelchips

- 20 g gerösteten Erdnüssen

- 20 g Schokolade

- 32 g Schlagsahne

- 4 Tassen Milchkaffee

Ein zu hoher Fettverzehr kann zu Übergewicht führen. Mit den sich daran meist anschließenden Diäten ist leider oft das Problem der Fehlernährung verbunden.

So werden z. B. immer wieder Diäten propagiert, die einen Fettverzehr ohne Begrenzung erlauben und dafür die Kohlenhydratzufuhr drosseln. Unter dem Gesichtspunkt lediglich der Gewichtsabnahme scheint das zunächst auch eine durchaus Erfolg versprechende Strategie zu sein. Denn die meisten Menschen essen ohne gleichzeitigen Konsum von
Kohlenhydraten ungern große Fettmengen, senken also auf diese Weise recht verlässlich ihre Energieaufnahme. Es kommt zu schnellen Anfangserfolgen, denen auch die Atkins-Diät ihre Popularität verdankte. Alle großen Studien zu diesem Thema zeigen jedoch, dass der Gewichtsverlust durch Diäten nur von den Kalorienbeschränkungen im Allgemeinen und deren jeweiliger Dauer abhängt. Abgesehen davon hat der Verzehr von zu viel gesättigten Fettsäuren den negativen Effekt, dass er unabhängig von der sonstigen Lebensgestaltung deutlich das Risiko für koronare Herzerkrankungen erhöht (Zong et al. 2016).

Gute Programme zur Gewichtsreduktion sind deshalb immer mit einer vernünftigen Ernährungsumstellung verbunden. Dazu gehört eine ausgewogene Ernährung mit reichlichem Verzehr von komplexen Kohlenhydraten z. B. in Gemüse, Obst oder Getreide. Diese sind Träger von Mineralstoffen, Spurenelementen, bioaktiven Pflanzenstoffen, Ballaststoffen und den meisten Vitaminen. Dabei ist zu beachten, dass anders als die komplexen Kohlenhydrate die einfachen Kohlenhydrate in Form von Zucker lediglich Energielieferanten sind. So empfiehlt auch die WHO, die Aufnahme von einfachen Kohlenhydraten z. B. aus Honig, Marmelade, süßen Backwaren, süßen Getränken, Schokolade, sonstigen Süßigkeiten oder versteckt in diversen Fertiggerichten bzw. Speisezutaten auf unter $10 \%$ des täglichen Energiebedarfs zu senken und eine Zuckersteuer von mindestens $20 \%$ einzuführen (2016). Zu beachten ist in diesem Zusammenhang, dass auf Lebensmitteletiketten ein hoher Zuckeranteil häufig mit Dutzenden weniger klaren Begriffen wie Oligofruktose, Stärkesirup, Saccharose, Raffinose, Malzextrakt, Maltose etc. umschrieben wird. 